\title{
Misophonia: a narrative review about an elusive neuropsychiatric condition
}

\author{
Francisco Coutinho, Isabel Brandão \\ Clínica de Psiquiatria e Saúde Mental - Centro Hospitalar Universitário de São João
}

Corresponding author: francisco.coutinho89@gmail.com

\section{Objectives}

To review the literature concerning the clinical picture, therapeutic approach and future research goals concerning misophonia

\section{Background and aims \\ Misophonia is a neurobehavioural syndrome defined by a heightened autonomic nervous system arousal and negative emotional reactivity towards specific sounds. It is important to make a differential diagnosis with other auditory conditions such as hiperaccusis or tinnitus.}

\section{Materials and methods}

Bibliographic search in Medline, with the keywords "misophonia" and "selective sound sensitivity syndrome"

\section{Results}

Despite being recently described, there are some proposed diagnostic criteria for misophonia, including those recently published by Dozier et al. There are still many doubts concerning both the etiology and theraupeutic approach of misophonia, as we highlight:

\section{Proposed diagnostic criteria for misophonia}

-The presence or anticipation of a specific sensory experience such as a sound, sight, or other stimulus, provokes an impulsive, aversive physical and emotional response which typically begins with irritation or disgust that may quickly become anger.

-The stimulus elicits an immediate physical reflex response. The physical response cannot always be identified, but the presence of an immediate physical response may be used to more clearly identify the condition as misophonia

-A moderate duration of the stimulus (e.g., 15 s) elicits general physiological arousal

-Dysregulation of thoughts and emotions with rare but potentially aggressive outbursts. Aggressive outbursts may be frequent in children

-The negative emotional experience is later recognized as excessive, unreasonable, or disproportionate

-The individual tends to avoid the misophonic situation, or if he/she does not avoid it, endures the misophonic stimulus situation with discomfort or distress.

The individual's emotional and physical experience, avoidance, and efforts to avoid cause significant distress or significant interference in the person's life

\section{Etiological hypothesis and associations with other syndromes}

Association with other neuropsychiatric conditions (obsessive-compulsive disorder, attention deficit and hyperactivity disorder, Autism spectrum disorders, X-fragile syndrome, Tourette syndrome)

Trigger sounds cause ativation of the anterior insular córtex Abnormal connectivity between anterior insular córtex and pre-frontal córtex, hipocampus and amygdala

\author{
Possible therapeuthic approaches of misophonia \\ Psychoeducation about the condition \\ Use of headphones and earplugs \\ Exposure and response prevention \\ Acceptance and commitment therapy \\ Pharmacologic treatment based on agents with \\ dopaminergic or serotoninergic action
}

\section{Conclusions}

There is currently little information concerning misophonia, both related to its clinical characteristics, neurobiological basis and therapeutic approach. In the future, it will be important to have a better charaterization of the symptoms among those who report impairment form misophonia and clarify whether it occurs as a specific symdrome or as part of other psychiatric disorders. Also, studies concerning psychotherapeutic and pharmacologic approaches with large samples will be necessary to define its preferencial approach. 